\title{
Epidemiologia temporal da queima das pontas das folhas da cebola em diferentes regimes de aplicação de fungicidas
}

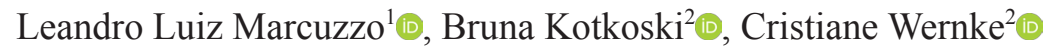

Instituto Federal Catarinense - IFC/Campus Rio do Sul, CP 441, CEP 89163-356, Rio do Sul, SC, Brasil. ${ }^{2}$ Aluna do curso de agronomia IFC/ Campus Rio do Sul, CP 441, CEP 89163-356, Rio do Sul, SC, Brasil. Bolsista PIBITI e PIBIC/Cnpq.

Autor para correspondência: Leandro Luiz Marcuzzo (leandro.marcuzzo@ifc.edu.br)

Data de chegada: 13/07/2017. Aceito para publicação em: 10/02/2019.

$10.1590 / 0100-5405 / 206334$

\section{RESUMO}

Marcuzzo, L.L.; Kotkoski, B.; Wernke, C. Epidemiologia temporal da queima das pontas das folhas da cebola em diferentes regimes de aplicação de fungicidas. Summa Phytopathologica, v.45, n.4, p.420-423, 2019.

A queima das pontas das folhas da cebola é controlada por meio de aplicação foliar com fungicidas seguindo um calendário fixo. Porém, o regime de aplicação baseado em um sistema de previsão, pode predizer e minimizar o progresso da doença. Como não se conhece o comportamento epidemiológico da doença nas condições do sul do Brasil, objetivou-se por meio desse trabalho, analisar a epidemiologia temporal da doença em diferentes regimes de aplicação de fungicidas. Em delineamento experimental de blocos casualizados com quatro repetições foram avaliados os regimes de aplicação baseado no sistema de Marcuzzo \& Haveroth (2016) com valores diários de severidade estimada (SE) acumulado de 0,20,0,25, e 0,30 comparados ao controle padrão (aplicação de fungicidas aos 5 e 7 dias). A avaliação da severidade da doença foi realizada semanalmente em dez plantas ao acaso previamente demarcadas em cada repetição. A curva de progresso da doença para cada um dos regimes foi ajustada ao modelo de Gompertz, já que a severidade observada correspondeu ao modelo e foi confirmada pela coerência entre os pontos estimados e pelo resíduo. Foi verificado que o regime de 7 dias apresentou a maior taxa $(0,80283)$ de progresso, enquanto que $\mathrm{SE}=0,30$ a menor $(0,50526)$. Verificou-se que o regime com $\mathrm{SE}=0,30$ apresentou o pico máximo da severidade com $46,4 \%$ e que o regime de 5 dias teve o menor acúmulo da doença com $27,7 \%$. Os regimes de aplicação de fungicida não influenciaram no comportamento do progresso temporal da queima das pontas das folhas da cebola Empasc 352/Bola Precoce.

Palavras-chave: Allium cepa, Botrytis squamosa, epidemiologia.

\section{ABSTRACT}

Marcuzzo, L.L.; Kotkoski, B; Wernke, C. Temporal epidemiology of Botrytis leaf blight of onion under different fungicide spray regimens. Summa Phytopathologica, v.45, n.4, p.420-423, 2019.

Botrytis leaf blight of onion is controlled by foliar fungicide application following a fixed schedule. However, the application regimen based on a forecast system can predict and minimize the disease progression. As the epidemiological behavior of the disease in southern Brazil is not known, this study aimed to analyze the temporal epidemiology of the disease under different fungicide application regimens. In a randomized block design with four replicates, the application regimens were evaluated based on the system of Marcuzzo \& Haveroth (2016) with cumulative daily values of estimated severity (ES) of $0.20,0.25$, and 0.30 , compared to the standard control (fungicide application at 5 and 7 days). The disease severity was weekly evaluated in ten plants previously demarcated at random for each replicate. The disease progress curve for each genotype was adjusted to Gompertz model, since the observed severity corresponded to the model and was confirmed by the consistency between the estimated points and by the residue. The 7 -day regimen had the highest progression rate $(0.80283)$, while $\mathrm{ES}=0.30$ had the lowest rate (0.50526). The regimen showing $\mathrm{ES}=0.30$ presented the maximum severity peak, $46.4 \%$, and the 5 -day regimen had the lowest cumulative value of the disease, $27.7 \%$. Fungicide application regimens did not influence the temporal progression of Botrytis leaf blight in onion Empasc 352/Bola Precoce.

Keywords: Allium cepa, Botrytis squamosa, epidemiology.

A cebola (Allium cepa L.) é cultivada em praticamente todo o Brasil, no entanto a ocorrência de doenças é um fator limitante para a produção da cultura. Entre as doenças, a queima das pontas das folhas causada por Botrytis squamosa Walker, tem sido considerada uma das mais destrutivas nas épocas de cultivo com temperaturas amenas $(\leq 22$ $\left.{ }^{\circ} \mathrm{C}\right)$ e alta umidade relativa do ar $(\geq 90 \%)(2,10)$.

$\mathrm{O}$ uso de diferentes regimes de aplicação pode ser uma das formas de manejo dessa doença, porém é necessária a avaliação do comportamento temporal da doença entre eles. As formas de caracterizar o desenvolvimento da doença, a curva de progresso temporal é a melhor representação de uma epidemia. A interpretação do formato dessas curvas e seus componentes, como a taxa e a severidade final são fundamentais para se efetuar o manejo de epidemias (3).

Como não se dispõe de informação sobre o assunto, o objetivo deste trabalho foi caracterizar o progresso temporal da queima das pontas das folhas da cebola em função de diferentes regimes de aplicação.

O experimento foi realizado entre 16 de abril e 29 de junho de 2018 no Instituto Federal Catarinense, Campus de Rio do Sul, município 

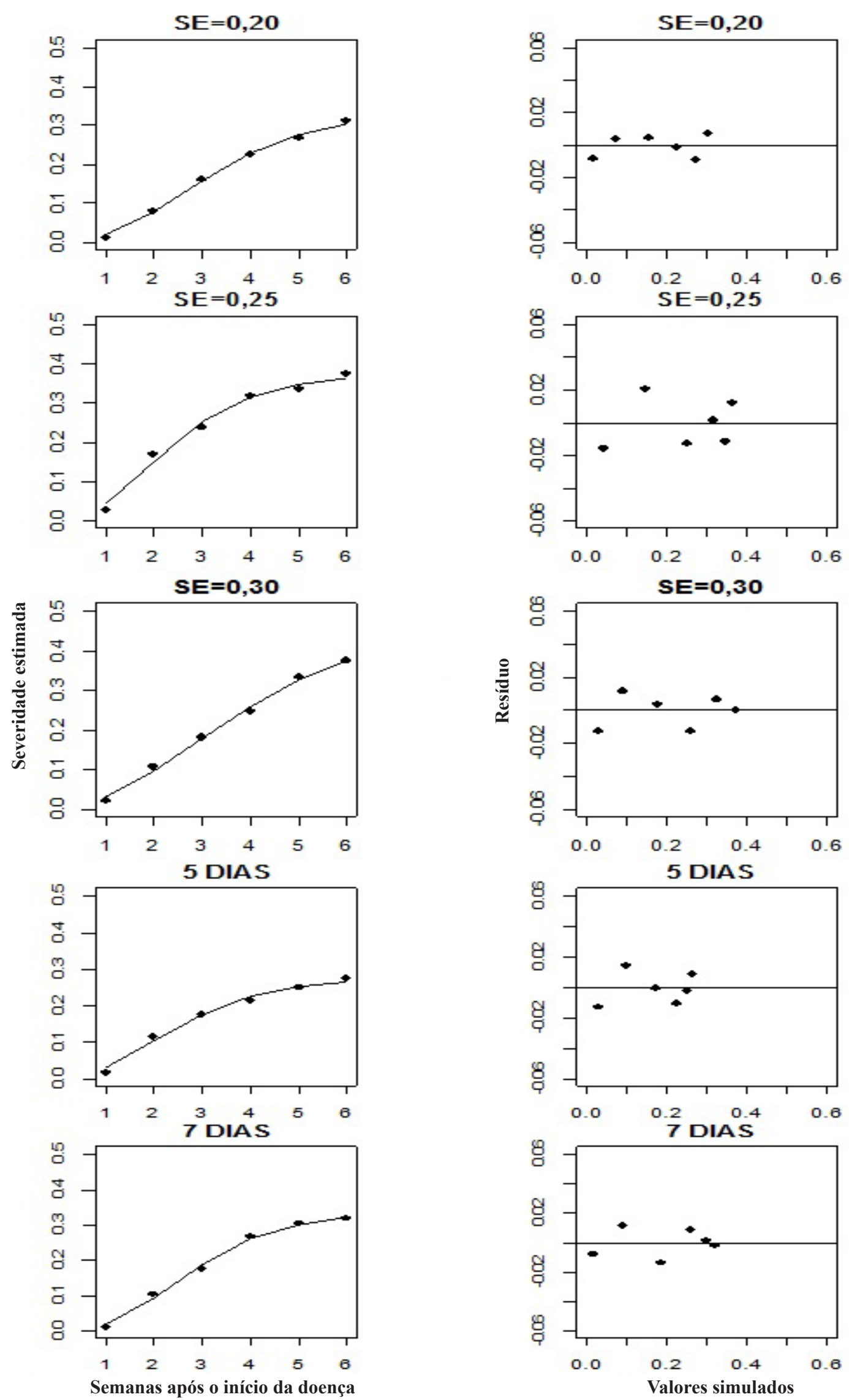

Figura 1. Curva da severidade estimada para o progresso da queima das pontas das folhas da cebola, cv. Empasc 352/Bola Precoce, sob diferentes regimes de aplicação de fungicidas, e seus respectivos resíduos ajustados pelo modelo de Gompertz. IFC/Campus Rio do Sul, 2018. 
Tabela 1. Parâmetros estimados pelo modelo de Gompertz ajustado às médias de queima das pontas das folhas da cebola cv. Empasc 352/Bola Precoce em diferentes regimes de aplicação de fungicida. IFC/Campus Rio do Sul, 2018

\begin{tabular}{|c|c|c|c|c|}
\hline \multirow[t]{2}{*}{ Regime de aplicação } & \multicolumn{4}{|c|}{ Parâmetros do modelo de Gompertz* } \\
\hline & Ymax & $\ln (\mathrm{y} 0 / \mathrm{ymax})$ & $\mathbf{r}$ & $R^{2^{* * *}}$ \\
\hline $\mathrm{SE}=0,25$ & 0,37534 & 5,08531 & 0,84785 & 0,98648 \\
\hline 5 DIAS & 0,27747 & 4,82544 & 0,78670 & 0,98748 \\
\hline 7 DIAS & 0,33849 & 6,52582 & 0,80283 & 0,99416 \\
\hline $\mathrm{CV}^{2}(\%)$ & & & 34,35 & \\
\hline
\end{tabular}

de Rio do Sul/SC, com latitude Sul de $27^{\circ} 11^{\prime} 07^{\prime \prime}$, longitude Oeste de 49³9’39" e altitude de 687 metros do nível do mar.

Os dados meteorológicos foram obtidos por meio de uma estação Davis $^{\circledR}$ Vantage Vue $300 \mathrm{~m}$ localizado ao lado do experimento e os dados médios durante a condução do experimento foram de $15,1^{\circ} \mathrm{C}$ para temperatura do ar, de 13,1 horas de umidade relativa do ar $\geq 90 \%$ e a precipitação pluvial acumulada de $171 \mathrm{~mm}$.

Três gramas de sementes de cebola da cultivar Empasc 352/Bola Precoce foram semeadas a campo em experimento constituído de blocos casualizados com cinco tratamentos e quatro repetições. Cada repetição apresentava uma área de 1,00 x 1,00 m, totalizando no mínimo de 600 plantas por repetição. Para avaliação da queima das pontas, dez plantas em cada parcela foram previamente escolhidas e demarcadas aleatoriamente. A calagem, adubação, assim como os tratos culturais realizados seguiram as normas da cultura (8) e não se utilizou inseticidas devido a não ocorrência de insetos no período de avaliação.

Para que houvesse inóculo do patógeno na área, mudas de cebola com 30 dias de idade foram inoculadas com auxílio de um atomizador portátil. Para isso, foi aplicada uma suspensão de conídios de B. squamosa $\left(10^{4}\right)$ obtida de folhas de cebola e multiplicada em meio de cultura BDA e, após 24 horas de câmara úmida, as mudas foram transplantadas a cada 1 metro linear ao redor do experimento no dia da semeadura. Também foram depositados cinco escleródios do patógeno produzidos pela técnica de Marcuzzo et al. (7) entre as parcelas, para servir como inóculo primário da doença na área.

Para o controle da queima das pontas foram comparados os seguintes regimes de aplicação com mancozeb $(80 \%)+$ oxicloreto de cobre (50\%) na dose de $250 \mathrm{~g}+200 \mathrm{~g} \mathrm{pc} \cdot \mathrm{hl}^{-1}$ baseado no modelo descrito por Marcuzzo \& Haveroth (6) expresso em SE $=0,008192 *(((x-$ $\left.\left.5)^{1,01089}\right) *\left((30-x)^{1,19052}\right)\right) *(0,33859 /(1+3,77989 * \exp (-0,10923 * y)))$, em que SE, representa o valor da severidade estimada $(0,1) ; x$, a temperatura $\left({ }^{\circ} \mathrm{C}\right)$ e $y$, o molhamento foliar (horas).

Atribuiu-se os tratamentos com valores acumulados de SE de 0,20; 0,25 , e 0,30 comparados com sistema convencional com aplicação a cada 5 dias e 7 dias. A aplicação no sistema de previsão foi realizada quando o somatório diário dos valores de SE $(020 ; 0,25 ; 0,30)$ fosse atingida, sendo então zerado o somatório e iniciada nova contagem dos valores de severidade diários.

As aplicações correspondentes a cada tratamento iniciaram-se aos 21 dias após a semeadura com um pulverizador costal eletrônico Jetbras $^{\circledR}$ calibrado para 400 L.ha $^{-1}$. A cada ocorrência de $25 \mathrm{~mm}$ de chuva, todos os tratamentos eram pulverizados, zerados e reiniciava-se a contagem do somatório dos valores de severidade.

A avaliação da severidade da queima das pontas foi realizada semanalmente após o início da doença (aos 30 dias após a semeadura), totalizando, portanto, 6 avaliações. A severidade da doença foi estimada visualmente determinando-se o percentual da área foliar necrosada pela doença em relação ao total ( 0 a 100\%) em cada folha presente na planta conforme metodologia utilizada por Boff et al. (4) e a incidência foi avaliada pelas folhas totais em relação às folhas doentes.

Modelos não lineares, comumente usados para representar crescimento de epidemias como o Logístico e o de Gompertz foram usados para ajuste com os dados observados utilizando o software $R$ versão 2.15 .1 (9). Os critérios estabelecidos para comparação dos modelos, em função da qualidade do ajustamento dos dados foram: a) erro padrão da estimativa; b) estabilidade dos parâmetros; c) erro padrão dos resíduos; d) visualização da distribuição dos resíduos ao longo do tempo e e) pseudo $\mathrm{R}^{2}$.

Os dados da taxa foram submetidos à análise de variância pelo teste $\mathrm{F}$, e se significativos, as médias seriam comparadas estatisticamente pelo teste de Tukey $(p \leq 0,05)$ pelo software estatístico SASM-Agri (5) para avaliar se houve diferença entre os regimes de aplicação quanto à taxa de progresso da doença.

O modelo de Gompertz expresso por $\mathrm{y}=\mathrm{ymax} *(\exp (-\ln (\mathrm{y} 0 / \mathrm{ymax})$ $* \exp (-\mathrm{r} * \mathrm{x})))$, onde $\mathrm{y}$ : severidade estimada final (\% de severidade $/ 100)$; ymax: severidade máxima de doença/100; $\ln (\mathrm{y} 0 / \mathrm{ymax})$ refere-se a função de proporção da doença na primeira observação; r: taxa, x o tempo em semanas após o início da doença foi escolhido para representar o progresso da queima das pontas na avaliação dos regimes de aplicação (Tabela 1), devido a queima das pontas ser considerada uma doença explosiva após o início dos primeiros sintomas. Possivelmente todos os dados se ajustaram em decorrência de ser a época de cultivo da cultura e a presença da doença no local.

A análise dos dados e as equações originadas pelo modelo de Gompertz (Tabela 1) resultaram em um coeficiente de determinação significativo, e a severidade observada correspondeu ao modelo, confirmada pela coerência entre os pontos estimados e do resíduo (erro) nas seis semanas de avaliação da doença (Figura 1).

A taxa de progresso da doença não apresentou diferenças estatísticas entre os regimes de aplicação (Tabela 1). Foi verificado que o regime com 7 dias apresentou a maior taxa $(0,80283)$ de progresso, enquanto que $\mathrm{SE}=0,30$ a menor $(0,50526)$. Constatou-se que o $\mathrm{SE}=0,30$ apresentou o pico máximo da severidade com $46,4 \%$ e que o regime de 5 dias teve o menor acúmulo da doença com $27,7 \%$, com uma diferença de $18,7 \%$ na severidade máxima entre esses dois regimes (Tabela 1).

Boff et al. (4) encontraram valores de severidade de $21,2 \%$ quando realizada a aplicação semanal com calda boldalesa $(0,5 \%)$, valor próximo ao verificado nesse trabalho (Figura 1), mas sem caracterizar o progresso da doença, mesmo quando foram avaliados diferentes ingredientes ativos de fungicidas, nas mesmas condições da região de 
execução desse trabalho (1).

Conclui-se que os regimes de aplicação de fungicidas avaliados não interferem no comportamento de progresso temporal da queima das pontas das folhas da cebola, causado pelo fungo Botrytis squamosa.

\section{REFERÊNCIAS}

1. Araújo, E.R.; Alves, D.P.; Higashikawa. Cultivar resistance and chemical, biological and fertilizer treatments for controling Botrytis leaf blight of onion. Tropical plant pathology, Brasília, v.43, n,2, p.160-164, 2018.

2. Araújo, E.R.; Marcuzzo, L.L.; Alves, D.P. Manejo de doenças. In: Nick, C.; Borém, A. Cebola: do plantio a colheita. Viçosa: UFV, Cap.7, p.126$155,2018$.

3. Bergamim Filho, A.; Amorim, L. Doenças de plantas tropicais: epidemiologia e controle econômico. São Paulo: Ceres, 1996, 289p.

4. Boff, P.; Gonçalves, P.A.S.; Debarba, J.F. Efeito de preparados caseiros no controle da queima-acizentada, na cultura da cebola. Horticultura brasileira, Brasília, DF, V.17, n.2, p.81-85, 1999.

5. Canteri, M.G.; Althaus, R.A.; Virgens Filho, J.S.; Giglioti, E.A.; Godoy, C.V.
SASM - Agri: Sistema para análise e separação de médias em experimentos agrícolas pelos métodos Scoft - Knott, Tukey e Duncan. Revista Brasileira de Agrocomputação, Ponta Grossa, v.1, p.18-24, 2001.

6. Marcuzzo, L.L.; Nascimento, A.; Kotkoski, B. Technique for inducing Botrytis squamosa sclerotium formation in vitro. Summa phytopathologica, Botucatu, v.43, n.3, p.251, 2017.

7. Marcuzzo, L.L.; Haveroth, R. Development of a weather-based model for Botrytis leaf blight of onion. Summa phytopathologica, Botucatu, v. 42 , n.1, p.92-93, 2016.

8. Menezes Júnior, F.O.G; Marcuzzo, L.L. Manual de boas práticas agrícolas: sustentabilidade das lavouras de cebola do estado de Santa Catarina. Florianópolis: Epagri, 2016, 143p.

9. R Development Core Team (2012). R: A language and environment for statistical computing. R Foundation for Statistical Computing, Vienna, Austria. Disponível em: <http://www.R-project.org $>$. Acesso em: 30 jun. 2018.

10. Wordell Filho, J. A.; Boff, P. Queima-acizentada - Botrytis squamosa Walker. In: Wordell Filho, J.A.; Rowe, E.; Gonçalves, P.A.; Debarba, J.F.; Boff, P.; Thomazelli, L.F.. Manejo Fitossanitário na cultura da cebola. Florianópolis: EPAGRI, p.19-30, 2006. 